\title{
Different Treatment Modalities of Colo-Rectal Cancer (Retrospective Study)
}

\author{
Abbas Mahmoud Sarhan', Mohammed Abdelgawad Soliman', \\ Eman Ali El-Sebai ${ }^{1}$, Mahmoud Mohammed Ahmed El-Demery*2 \\ ${ }^{1}$ Department of Clinical Oncology, Faculty of Medicine, Zagazig University, Sharkia, Egypt. \\ ${ }^{2}$ Department of Clinical Oncology \& Nuclear Medicine, Ahmed Maher Teaching Hospital, Cairo, Egypt \\ *Corresponding Author: Mahmoud Mohammed Ahmed El-Demery, E-mail: mahmod.eldemery@ gmail.com,
}

Mobile: (+20)01222922230

\begin{abstract}
Background: Colorectal Cancer (CRC) is the most common type affecting the gastrointestinal tract accounting for about $13 \%$ of all malignancy diagnosed each year. Although Surgery stays the most effective treatment in managing colorectal carcinoma, there is a rapid increase in the use of preoperative radiotherapy and chemotherapy.

Objective: So, the present study was conducted to identify the different therapeutic modalities used in the treatment. Patients and Methods: This study was a retrospective study that included CRC patients treated at the Clinical Oncology Department, Zagazig University Hospital during the period from 2012 to 2017. Medical records of 385 patients, who were referred to the Clinical Oncology Department after being diagnosed with CRC were obtained and used to collect data about different treatments and then analyzed.

Results: Chemotherapy regimen FOLFOX was received in (45.5\%) of cases and partial response in $41 \%$ of cases was observed. Patients received at least one line of chemotherapy with either adjuvant or palliative intent. Rectal cancer patients who received neoadjuvant concurrent chemo\& radiotherapy showed a partial response in $45.5 \%$ of cases. Xeloda was the most common chemotherapy received in most cases who received either adjuvant or neoadjuvant CCRT. Recurrence occurred in $(81 \%)$ of cases.

Conclusion: Chemotherapy regimen FOLFOX was received in most cases. Patients received at least one line of chemotherapy with either adjuvant or palliative intent, FOLFOX was the most chosen protocol. Xeloda was the most common chemotherapy received in most cases.
\end{abstract}

Keywords: treatment modalities, Colorectal Cancer, Retrospective.

\section{INTRODUCTION}

Colorectal Cancer (CRC) is the most common cancer affecting the gastrointestinal tract. About twothirds of all colorectal cancers were presented in the developed regions of the world. It also comes as the second leading cause of cancer-related deaths. It is the third most diagnosed cancer in males and the second in females, with 1.8 million new cases and almost 861,000 deaths in 2018 according to the World Health Organization ${ }^{(\mathbf{1})}$.

In Egypt, the average number of colon cancer patients (without rectal cancer) in 2015 was slightly more than three thousand ${ }^{(2)}$. Evaluation of the entire colon \& rectum and accurate staging are essential for optimal treatment of colorectal cancers. The TNM staging system is the main tool used for CRC staging (3).

Surgery stays the only curative and most effective treatment in managing colorectal carcinoma. But in the last decade, there is a rapid increase in the use of preoperative radiotherapy, neoadjuvant, and adjuvant chemotherapy ${ }^{(4)}$.

The liver is the most common site of distant metastases from colorectal cancer with approximately $25 \%$ of patients diagnosed with synchronous colorectal liver metastases (CLM) at presentation. Surgical resection is the current treatment of choice for colorectal cancer metastases isolated to the liver and has been proven to be the only potentially curative therapy ${ }^{(5)}$.

Metastatic irresectable CRC patients usually receive either palliative chemotherapy, radiotherapy, or BSC (Best Supportive Care) according to their performance status, tolerance to treatment ${ }^{\left({ }^{(6)}\right.}$.

Chemotherapy is routinely added to long-course radiation treatment as chemo-radiation was associated with a lower risk of local recurrence and improved disease-free survival, compared with radiation treatment alone ${ }^{(7)}$. So, the present study was conducted to identify the different therapeutic modalities used in CRC and their impact on patients' outcomes.

\section{PATIENTS AND METHODS}

This was a retrospective study on CRC patients treated at the Clinical Oncology Department, Zagazig University Hospital during the period from 2012 to 2017. The medical records of 385 patients, who were referred to the department after a proven diagnosis with CRC were enrolled in the study. A detailed history and clinical examination were revised. The intent of management: whether curative or palliative was addressed.

Chemo and Radiotherapy setting whether received in adjuvant or neoadjuvant setting data was collected and each group had been subdivided into smaller 
groups depending on chemotherapy protocol and modality of radiation.

Response to therapy (in case of neoadjuvant therapy) was assessed then patients who received neoadjuvant therapy were divided into four groups according to their response to therapy: complete response, partial response, stable disease, and progression. Recurrence pattern and time of recurrence was documented.

\section{Ethical Considerations:}

The study was approved by the Ethics Board of Zagazig University and an informed written consent was taken from each participant in the study.

\section{Statistical Analysis}

All data were collected, tabulated, and statistically analyzed using SPSS 20.0 for windows (SPSS Inc., Chicago, IL, USA). Quantitative variables were expressed as the mean $\pm \mathrm{SD}$ and the categorical variables were expressed as a number and percentage.

\section{RESULTS}

The whole number of CRC cases diagnosed, treated at the Clinical Oncology Department, Zagazig University Hospital during the chosen period was found 385 cases with the highest incidence of $19.2 \%$ was in 2017.

\section{Chemotherapy setting:}

Table (1), showed treatment data of 33 cases, with colon cancer who received neoadjuvant chemotherapy. Chemotherapy regimen FOLFOX was received in most cases $(45.5 \%)$, clinical response was assessed in 12 cases, showing partial response in $41 \%$ of cases.

Also, 181 patients received at least one line of chemotherapy with either adjuvant or palliative intent, FOLFOX was the most common chosen protocol in (45.9\%) of cases. As seen in Table 2.

\section{Concurrent chemo\& radiotherapy setting:}

Table 3 showed treatment data of 57 cases, with rectal cancer who received neoadjuvant concurrent chemo\& radiotherapy, clinical response was assessed in 11 cases, showing partial response in $45.5 \%$ of cases. xeloda was the most common chemotherapy received in most cases who received either adjuvant or neoadjuvant CCRT (63.2\%) in neoadjuvant CCRT \& (41.7\%) in adjuvant CCRT Table 4.

\section{Treatment Outcome:}

Table 5 showed outcome data of 100 cases, local recurrence was observed in $(81 \%)$ of cases, about $(59 \%)$ of patients developed distant metastasis during follow-up after surgery $+/$ - adjuvant or neoadjuvant therapy.

Table (1): Received neoadjuvant chemotherapy in the studied colorectal carcinoma patients.

\begin{tabular}{|c|c|c|}
\hline \multirow{2}{*}{ Received neoadjuvant chemotherapy } & \multicolumn{2}{|c|}{ All patients } \\
\cline { 2 - 3 } & \multicolumn{2}{|c|}{ No. } \\
\hline Clinical response & 5 & $41.7 \%$ \\
\hline Partial response & 4 & $33.3 \%$ \\
\hline Stable disease & 3 & $25 \%$ \\
\hline Progressive disease & & \\
\hline
\end{tabular}

Table (2): Comparison between adjuvant and palliative chemotherapy among the studied colorectal carcinoma patients.

\begin{tabular}{|c|c|c|c|c|c|c|}
\hline \multirow[t]{2}{*}{ Regimen } & \multicolumn{2}{|c|}{$\begin{array}{c}\begin{array}{c}\text { Adjuvant } \\
\text { chemotherapy } \\
(\mathrm{N}=142)\end{array} \\
\end{array}$} & \multicolumn{2}{|c|}{$\begin{array}{c}\text { Palliative } \\
\text { chemotherapy } \\
(\mathrm{N}=39)\end{array}$} & \multicolumn{2}{|c|}{$\begin{array}{l}\text { Neo-adjuvant } \\
\text { chemotherapy } \\
(\mathrm{N}=33)\end{array}$} \\
\hline & No. & $\%$ & No. & $\%$ & No. & $\%$ \\
\hline 5FU-Leu & 40 & $28.2 \%$ & 5 & $12.8 \%$ & 7 & $21.2 \%$ \\
\hline FOLOFX & 68 & $47.9 \%$ & 17 & $43.6 \%$ & 15 & $45.5 \%$ \\
\hline XELOX & 12 & $8.5 \%$ & 6 & $15.4 \%$ & 8 & $24.5 \%$ \\
\hline Xeloda & 15 & $10.6 \%$ & 2 & $5.1 \%$ & 3 & $9.1 \%$ \\
\hline FOLFIRI & 6 & $4.2 \%$ & 8 & $20.5 \%$ & 0 & 0 \\
\hline Other & 1 & $0.7 \%$ & 1 & $2.6 \%$ & & \\
\hline
\end{tabular}


Table (3): Received concurrent chemoradiation in the studied colorectal carcinoma patients.

\begin{tabular}{|c|c|c|}
\hline \multirow[b]{2}{*}{ Received concurrent chemoradiation } & \multicolumn{2}{|c|}{ All patients } \\
\hline & No. & $\%$ \\
\hline Setting & \multicolumn{2}{|c|}{$(\mathrm{N}=117)$} \\
\hline Adjuvant & 60 & $51.3 \%$ \\
\hline Neoadjuvant & 57 & $48.7 \%$ \\
\hline Chemotherapy regimen & \multicolumn{2}{|c|}{$(\mathrm{N}=117)$} \\
\hline 5FU-Leu & 55 & $47 \%$ \\
\hline Xeloda & 61 & $52.1 \%$ \\
\hline Cisplatin & 1 & $0.9 \%$ \\
\hline Radiotherapy modality & \multicolumn{2}{|c|}{$(\mathrm{N}=117)$} \\
\hline $2 \mathrm{D}$ & 84 & $71.8 \%$ \\
\hline $3 \mathrm{D}$ & 33 & $28.2 \%$ \\
\hline Radiotherapy dose: & \multicolumn{2}{|c|}{$(\mathrm{N}=117)$} \\
\hline $45 \mathrm{~Gy}-1.8 \mathrm{~Gy} / \mathrm{f}$ & 92 & $78.6 \%$ \\
\hline $50 \mathrm{~Gy}-2 \mathrm{~Gy} / \mathrm{f}$ & 25 & $21.4 \%$ \\
\hline Clinical response & \multicolumn{2}{|c|}{$(\mathrm{N}=11)$} \\
\hline Complete response & 2 & $18.2 \%$ \\
\hline Partial response & 5 & $45.5 \%$ \\
\hline Stable disease & 3 & $27.3 \%$ \\
\hline Progressive disease & 1 & $9.1 \%$ \\
\hline
\end{tabular}

Table (4): Comparison between adjuvant and neoadjuvant concurrent chemoradiation sitting among the studied rectal carcinoma patients.

\begin{tabular}{|c|c|c|c|c|}
\hline \multirow[t]{2}{*}{ the } & \multicolumn{2}{|c|}{ Adjuvant sitting } & \multicolumn{2}{|c|}{ Neoadjuvant sitting } \\
\hline & No. & $\%$ & No. & $\%$ \\
\hline Chemotherapy regimen & \multicolumn{2}{|c|}{$(\mathrm{N}=60)$} & \multicolumn{2}{|c|}{$(\mathrm{N}=57)$} \\
\hline 5FU-Leu & 34 & $56.7 \%$ & 21 & $36.8 \%$ \\
\hline Xeloda & 25 & $41.7 \%$ & 36 & $63.2 \%$ \\
\hline Cisplatin & 1 & $1.7 \%$ & 0 & $0 \%$ \\
\hline Radiotherapy modality & \multicolumn{2}{|c|}{$(\mathrm{N}=59)$} & \multicolumn{2}{|c|}{$(\mathrm{N}=57)$} \\
\hline $2 \mathrm{D}$ & 44 & $74.6 \%$ & 40 & $70.2 \%$ \\
\hline $3 \mathrm{D}$ & 15 & $25.4 \%$ & 17 & $29.8 \%$ \\
\hline Radiotherapy dose: & \multicolumn{2}{|c|}{$(\mathrm{N}=60)$} & \multicolumn{2}{|c|}{$(\mathrm{N}=57)$} \\
\hline $45 \mathrm{~Gy}-1.8 \mathrm{~Gy} / \mathrm{f}$ & 41 & $68.3 \%$ & 51 & $89.4 \%$ \\
\hline $50 \mathrm{~Gy}-2 \mathrm{~Gy} / \mathrm{f}$ & 19 & $31.7 \%$ & 6 & $10.6 \%$ \\
\hline
\end{tabular}

Table (5): Outcome of the studied colorectal carcinoma patients.

\begin{tabular}{|c|c|c|}
\hline \multirow{2}{*}{ Outcome } & \multicolumn{2}{|c|}{$\begin{array}{c}\text { All patients } \\
(\mathbf{N = 1 0 0})\end{array}$} \\
\cline { 2 - 3 } & No. & \% \\
\hline Local failure & & $81 \%$ \\
\hline Absent & 81 & $19 \%$ \\
\hline Present & 19 & \\
\hline Regional failure & & $81 \%$ \\
\hline Absent & 81 & $19 \%$ \\
\hline Present & 19 & \\
\hline Distant metastasis & & $59 \%$ \\
\hline Absent & 59 & $41 \%$ \\
\hline Present & 41 & \\
\hline
\end{tabular}




\section{DISCUSSION}

Colorectal cancer (CRC) is a major cause of morbidity and mortality worldwide. It is the third most common cancer with 1.4 million new cases in 2012 and the fourth-leading cause of cancer with 693,333 deaths each year in the world ${ }^{(8)}$.

Surgery is the only curative treatment for CRC. About half of CRC patients develop the metastatic disease with no curative treatment except in the minority of patients. Survival can be improved with palliative chemotherapy regimens ${ }^{(9)}$.

This study was conducted in the Clinical Oncology and Nuclear Medicine Department in Zagazig University Hospitals including all old CRC patients who attended the outpatient clinic and received either form of treatment (surgery, preoperative neoadjuvant therapy, adjuvant therapy, or palliative chemotherapy/radiotherapy (RT)) from 2012 to 2017.

Neoadjuvant treatment with radiation (with or without chemotherapy), followed by surgery, is current practice for managing most mid-low rectal cancers that are staged preoperatively as at least T3 and/or at least N1 (i.e. Stage II or III), in individuals well enough to tolerate it, also for a limited group of patients with $\mathrm{T} 4$ colon cancers penetrating the fixed structure. The timing of treatment preoperatively rather than postoperatively is based on the results of the CAO/ARO/AIO-94 study, a serial 2004 phase III randomized controlled trial comparing preoperative with postoperative chemoradiation, which reported a significant improvement in local control in favor of neoadjuvant chemoradiation. This finding changed practice at the time ${ }^{(\mathbf{1 0})}$.

The present study shows that treatment data of 33 cases, with colon cancer who received neoadjuvant chemotherapy, chemotherapy regimen FOLFOX was received in most cases (45.5\%). Clinical response was assessed in 12 cases, showing partial response in $41 \%$ of cases. treatment data of 57 cases, with rectal cancer who received neoadjuvant concurrent chemo\& radiotherapy, clinical response was assessed in 11 cases, showing partial response in $45.5 \%$ of cases. xeloda was the most common chemotherapy received in most cases who received either adjuvant or neoadjuvant CCRT (63.2\%) in neoadjuvant CCRT \& (41.7\%) in adjuvant CCRT.

Randomized clinical trials demonstrated that neoadjuvant chemoradiotherapy reduces local recurrence in lower rectal cancer ${ }^{(11)}$. It was given in the study of Telfah et al. ${ }^{(12)}$ to 38 patients of these 5 patients developed complete response at the histopathology report.

There is no doubt that adjuvant chemotherapy prolongs time to disease progression and overall survival when compared to best supportive care, adjuvant chemotherapy was given in most nonmetastatic CRC, and palliative chemotherapy was given in also most patients with metastatic disease ${ }^{(\mathbf{1 3})}$.
Several studies have investigated the outcomes of colorectal disease in younger patients. Studies that compared the stage-to-stage survival of younger versus older patients with CRC found that younger patients with Duke A or B tumors appear to have better survival than older patients with the similar-stage disease (14). Perhaps this is secondary to better tolerance of surgery and adjuvant therapy by the younger patients.

Regarding Characteristics of postoperative histopathology, in the study in our hands, adenocarcinoma was observed in $(93.8 \%)$ of cases (79.3\%) of cases were grade II, the negative margin was noticed in $(93.8 \%)$ of cases. Fifty percent of patients were node-negative by operation pathology, while $50 \%$ of patients were of pathological stage III .181 patients received at least one line of chemotherapy with either adjuvant or palliative intent, FOLFOX was the most common chosen protocol in (45.9\%) of cases.

The present study results are supported by the study of Saha et $\mathbf{a l} .^{(15)}$ as they reported that all but two were adenocarcinoma histopathologically and the remaining two involving anal canal were squamous cell carcinoma.

Telfah et al. ${ }^{(12)}$ found that most of the tumors in their patients were moderate to high grade that means aggressive of this type of tumor and the need for adjuvant treatment after surgery. And only a few tumors were well-differentiated or grade 1 tumor. This finding is related partially to the late presentation of patients with CRC and delay of diagnosis.

Adjuvant chemotherapy post-surgery improves survival especially in Stage II and III. Delay to start adjuvant treatment affects the outcome ${ }^{(16)}$.

As regard outcome data of 100 cases, our results show that local recurrence was observed in $(81 \%)$ of cases, about $(59 \%)$ of patients developed distant metastasis during follow-up after surgery + /- adjuvant or neoadjuvant therapy.

Our results are supported by a study of Telfah $\boldsymbol{e t}$ al. (12) as they reported that all patients with nonmetastatic CRC were kept on regular follow-up at oncology clinic and included, carcinoembryonic antigen levels, abdominal and pelvic CT scan; about $70 \%$ of patients on regular follow up developed recurrence of disease, most common sites of recurrence were lymph nodes, liver, and lungs, which were the same common sites of metastasis at presentation. The high rate of recurrence was in T4 lesions and poorly differentiated tumors. The recurrence patterns of colon cancer and rectal cancer are quite different from more lung metastasis in rectal cancer. This finding is similar to recent studies in the USA $^{(17)}$.

The study of Chou et al. ${ }^{(18)}$ demonstrated that the overall survival rate of the younger group decreased dramatically in the first 3 years. A significant proportion of initial metastasis at diagnosis and rapid 
progression of advanced disease resulted in a marked decrease in the overall survival of the younger group in the first 3 years. The process of aging, underlying morbidities, and progression of CRC resulted in a slowly decreasing survival plot of elderly patients. Finally, the survival plots of the 2 study groups became very similar after the third year, and there was no difference in the overall survival rate between the 2groups (P 0.247). They observed that younger patients had significantly poor disease-free survival and cancer-specific survival. Cancer stage and emergency surgery were independent prognostic factors in the multivariate analysis of disease-free survival and cancer-specific survival. This means that poor disease-free survival and cancer-specific survival in younger patients were correlated with advanced nature and late presentation symptoms of the disease. In an attempt to further evaluate whether this different prognosis is due to a more advanced stage at presentation, they constructed TNM-specific survival curves for the 2 cohorts of patients. They combined stages I and II as the early stage and stages III and IV as the advanced stage. Different prognosis between the 2 cohorts of patients was due to a more advanced stage at presentation ( $\mathrm{P} 0.001)$.

\section{CONCLUSION}

Chemotherapy regimen FOLFOX was received in most cases. Also, 181 patients received at least one line of chemotherapy with either adjuvant or palliative intent. Xeloda was the most common chemotherapy received in most cases who received either adjuvant or neoadjuvant CCRT.

\section{REFERENCES}

1. Siegel R, Miller K, Fedewa S et al. (2017): Colorectal cancer statistics, 2017. A Cancer Journal for Clinicians, 67(3): 177-193.

2. Arnold M, Sierra M, Laversanne M et al. (2017): Global patterns and trends in colorectal cancer incidence and mortality. Gut, 66(4): 683-691.

3. Bidoli E, Serraino D (2017): Epidemiology of Cancer: Prevalence, Incidence of Neoplastic Diseases, and Trends in Survival in Europe. In Manual of Cardiooncology, Springer, Cham. Pp. 3-14.

4. Leongito M (2016): 7. Neoadjuvant folfiri+ bevacizumab in patients with resectable liver metastases from colorectal cancer: Results from a phase 2 trial and comparative analysis of early PET/CT scan vs RECIST criteria in predicting outcome. European Journal of Surgical Oncology, 42(9): 68-73.

5. Van Cutsem E, Lenz H, Köhne C et al. (2015): Fluorouracil, leucovorin, and irinotecan plus cetuximab treatment and RAS mutations in colorectal cancer. Journal of Clinical Oncology, 33(7): 692-700.

6. Engelhardt E, Révész D, Tamminga H et al. (2018): Clinical usefulness of tools to support decision-making for palliative treatment of metastatic colorectal cancer: a systematic review. Clinical Colorectal Cancer, 17(1): $1-12$.

7. Montroni I, Ugolini G, Saur N et al. (2018): Personalized management of elderly patients with rectal cancer: expert recommendations of the European Society of Surgical Oncology, European Society of Coloproctology, the International Society of Geriatric Oncology, and American College of Surgeons Commission on cancer. European Journal of Surgical Oncology, 44(11): 1685-1702.

8. Alwan A (2011): Global status report on noncommunicable diseases 2010. www.who.int/nmh/publications/ncd report 2010/ en/.

9. Pistoia V, Morandi F, Wang X et al. (2007): Soluble HLA-G: Are they clinically relevant? Semin Cancer Biol., 17:469-79.

10. Sauer R, Becker H, Hohenberger W et al. (2004): Preoperative versus postoperative chemoradiotherapy for rectal cancer. N Engl J Med., 351(17):1731-40.

11. Graham R, Wang S, Catalano $P$ et al. (1998): Postsurgical surveillance of colon cancer: Preliminary cost analysis of physician examination, carcinoembryonic antigen testing, chest $\mathrm{x}$-ray, and colonoscopy. Ann Surg., 228:59-63.

12. Telfah A, Obeidat M, Abu-Kamar A, Bawa'neh A et al. (2015): Clinicopathological characteristics of colorectal cancers at KHMC. Appl Med Res., 1(1): 2225

13. Buyse M, Piedbois P (2001): Should Dukes'B patients receive adjuvant therapy? A statistical perspective. Semin Oncol., 28:20-24.

14. Turkiewicz D, Miller B, Schache D et al. (2001): Young patients with colorectal cancer: how do they fare? ANZ J Surg., 71:707-10.

15. Saha M, Shil B, Saha S et al. (2016): Study of Clinicopathological Profile of Sporadic Cases of Colorectal Cancer. Euroasian Journal of HepatoGastroenterology, 6(2): 134-38.

16. Sanoff H, Carpenter W, Martin C et al. (2012): Comparative effectiveness of oxaliplatin vs nonoxaliplatin-containing adjuvant chemotherapy for stage III colon cancer. J Natl Cancer Inst., 104:211-27.

17. Sargent D, Goldberg R, Jacobson S et al. (2001): A pooled analysis of adjuvant chemotherapy for resected colon cancer in elderly patients. $\mathrm{N}$ Engl J Med., 345:1091-7.

18. Chou C, Chang S, Lin T et al. (2011): Differences in clinicopathological characteristics of colorectal cancer between younger and elderly patients: an analysis of 322 patients from a single institution. The American Journal of Surgery, 202(5): 574-582. 